Real Analysis Exchange

Vol. 23(2), 1997-1998, pp. 787-797

Marek Balcerzak, Institute of Mathematics, Łódź Technical University, al. Politechniki 11, 90-924 Lódź, Poland, e-mail: mbalce@krysia.uni.lodz.pl Krzysztof Ciesielski*, Department of Mathematics, West Virginia University, Morgantown, WV 26506-6310, USA, e-mail: KCies@wvnvms. wvnet. edu

\title{
ON THE SUP-MEASURABLE FUNCTIONS PROBLEM
}

\begin{abstract}
We show some results connected with the problem whether it is consistent that every sup-measurable function $F: \mathbb{R}^{2} \rightarrow \mathbb{R}$ is measurable. We will also relate this problem to a von Weizsäcker problem concerning a generalization of Blumberg theorem.
\end{abstract}

We use standard set-theoretical notation as in [3]. Symbol $|X|$ will stand for the cardinality of a set $X$. The cardinality of the set $\mathbb{R}$ of real numbers is denoted by $\mathfrak{c}$. For a set $E \subseteq \mathbb{R}^{2}$, we denote by $\operatorname{dom}(E)$ and $\operatorname{ran}(E)$ its projections on the first and on the second axis, respectively. A function $f$ from a subset of $\mathbb{R}$ into $\mathbb{R}$ will be identified with its graph. If $\mathcal{I}$ is a proper ideal of subsets of $\mathbb{R}$, by non $(\mathcal{I})$ we denote the minimal cardinality of a set that is not in $\mathcal{I}$. Symbols $\mathcal{M}$ and $\mathcal{N}$ will stand for the ideals of meager sets and of null sets in $\mathbb{R}$, respectively.

We say that a function $F: \mathbb{R}^{2} \rightarrow \mathbb{R}$ is sup-measurable if the function $F_{f}: \mathbb{R} \rightarrow \mathbb{R}$ given by $F_{f}(x)=F(x, f(x)), x \in \mathbb{R}$, is measurable for each measurable function $f: \mathbb{R} \rightarrow \mathbb{R}$. We will also consider a dual category analog notion that is obtain from the above by replacing the requirement of measurability of functions with the requirement that the appropriate functions have the Baire property. If we want to distinguish these two notions, we will use the phrases "Lebesgue sup-measurability" and "Baire sup-measurability." Observe that if in the above definitions we require that $F_{f}$ is measurable (or

\footnotetext{
Key Words: Lebesgue measurability, Baire property, sup-measurable function.

Mathematical Reviews subject classification: Primary: 26B30; Secondary: 04A15, 28A05, 54H05.

Received by the editors December 1, 1997

* This work was partially supported by NSF Cooperative Research Grant INT-9600548 with its Polish part financed by KBN.

Papers authored or co-authored by a Contributing Editor are managed by a Managing Editor or one of the other Contributing Editors.
} 
has the Baire property) only for every Borel function $f$ then we will still obtain the same notions. (See [1, Lemma 1.1].)

It is not hard to find measurable functions which are not sup-measurable. (See [13] or [1, Cor. 1.4].) Under the continuum hypothesis $\mathrm{CH}$ or some weaker set-theoretical assumptions, nonmeasurable sup-measurable functions were constructed in [7], [8], [9], and [1]. (Similarly, for the category case. See [6].) These rather exotic functions are called "monsters" in the literature. (See [10].) It is interesting to know whether the existence of such functions can be proved in ZFC or whether there is a model of ZFC with no such examples. (See [8], [9], [1], and [4] where this question was mentioned.) In our article we discuss these problems.

Note that the existence of nonmeasurable sup-measurable functions has some consequences in the theory of ordinary differential equations, which was described in detail in the recent article of Kharazishvili [9]. Namely, let $G: \mathbb{R}^{2} \rightarrow \mathbb{R}$ and $\left\langle x_{0}, y_{0}\right\rangle \in \mathbb{R}^{2}$. We say that the Cauchy problem

$$
y^{\prime}=G(x, y), \quad y\left(x_{0}\right)=y_{0}
$$

has a (unique) solution in the class $\mathrm{AC}_{l}$ of locally absolutely continuous functions on $\mathbb{R}$ if there exists a (unique) function $f \in \mathrm{AC}_{l}$ such that $f\left(x_{0}\right)=y_{0}$ and $f^{\prime}(x)=G(x, f(x))$ for almost all $x \in \mathbb{R}$. (Recall that $f \in \mathrm{AC}_{l}$ if each $x \in \mathbb{R}$ has a neighborhood $V$ such that the restriction $f \mid V$ of $f$ to $V$ is absolutely continuous.) We will describe several nonmeasurable (sup-measurable) functions $G$ for which (1) has a unique locally absolutely continuous solution.

In [1, Prop. 1.7] the author constructs (in ZFC) a nonmeasurable set $H \subseteq \mathbb{R}^{2}$ such that the characteristic function $F$ of $H$ satisfies the condition

$$
|\{x \in \mathbb{R}: F(x, f(x)) \neq 0\}|<\mathfrak{c}
$$

for every Borel function $f: \mathbb{R} \rightarrow \mathbb{R}$. Then $F$ is nonmeasurable and its supmeasurability is implied by non $(\mathcal{N})=\mathfrak{c}$. Consider the Cauchy problem (1) where $G(x, y)=F(x, y)+g(x)$ and $g: \mathbb{R} \rightarrow \mathbb{R}$ is a fixed locally Lebesgue integrable function. Note that $G$ is nonmeasurable. Using $\operatorname{non}(\mathcal{N})=\mathfrak{c}$ and (2) we can easily check that the function $\varphi(x)=y_{0}+\int_{x_{0}}^{x} g(t) d t, x \in \mathbb{R}$, is a locally absolutely continuous solution of (1). This solution is unique since if $\psi \in \mathrm{AC}_{l}$ is an arbitrary solution of (1) then $\psi\left(x_{0}\right)=y_{0}$ and

$$
\psi^{\prime}(x)=F(x, \psi(x))+g(x)
$$

for almost all $x$. But then $F_{\psi}$ is measurable. So the set $\left\{x \in \mathbb{R}: F_{\psi}(x) \neq 0\right\}$ is measurable and, by (2), it is of measure zero. Hence $F_{\psi}$ equals zero almost 
everywhere and from (3) we infer that

$$
\psi(x)=y_{0}+\int_{x_{0}}^{x} g(t) d t=\varphi(x)
$$

for every $x \in \mathbb{R}$. Note that in that last argument we do not use any extra set-theoretical assumptions. Also the whole power of sup-measurability of $F$ is not needed to obtain the solution $\varphi$ of (1). In fact, it suffices to know that $F_{f}$ equals zero almost everywhere for every Borel function $f$, which is implied by $\operatorname{non}(\mathcal{N})=\mathfrak{c}$.

The article [9] contains a construction similar to that from [1] but its advantage appears in special problems of type (1). Namely, in [9, Th.3] the respective nonmeasurable set $H \subseteq \mathbb{R}^{2}$ additionally meets every straight line in at most two points. In that case

$$
|\{x \in \mathbb{R}: F(x, f(x)) \neq 0\}| \leq 2
$$

provided that $F$ is the characteristic function of $H$ and $f$ is of the form $f(x)=$ $a x+b, x \in \mathbb{R}$. Thus the Cauchy problem (1), where $G(x, y)=F(x, y)+a$, has the unique locally absolutely continuous solution $\varphi(x)=a x+\left(y_{0}-a x_{0}\right)$, $x \in \mathbb{R}$, and this (by (4) and the previous argument) can be proved in ZFC. (See [9, Th.4].) However, we do not know whether this $F$ is sup-measurable. (Though it follows from $(2)$ and $\operatorname{non}(\mathcal{N})=\mathfrak{c}$.)

Now, let us recall the following simple lemma.

Lemma 1. [1, Prop. 1.5] The following conditions are equivalent:

(I) there is a nonmeasurable sup-measurable function $F: \mathbb{R}^{2} \rightarrow \mathbb{R}$;

(II) there is a nonmeasurable set $H \subseteq \mathbb{R}^{2}$ such that $\operatorname{dom}(H \cap f)$ is measurable for each Borel function $f: \mathbb{R} \rightarrow \mathbb{R}$;

(III) there is a nonmeasurable sup-measurable function $F: \mathbb{R}^{2} \rightarrow\{0,1\}$.

Remark 1. We can add three more conditions in Lemma 1:

$\left(\mathbf{I}^{\prime}\right)$ there is a nonmeasurable function $F: \mathbb{R}^{2} \rightarrow \mathbb{R}$ such that $F_{f}$ is measurable for each continuous function $f: \mathbb{R} \rightarrow \mathbb{R}$;

(II') there is a nonmeasurable set $H \subseteq \mathbb{R}^{2}$ such that $\operatorname{dom}(H \cap f)$ is measurable for each continuous function $f: \mathbb{R} \rightarrow \mathbb{R}$;

$\left(\mathbf{I I I}^{\prime}\right)$ there is a nonmeasurable function $F: \mathbb{R}^{2} \rightarrow\{0,1\}$ such that $F_{f}$ is measurable for each continuous function $f: \mathbb{R} \rightarrow \mathbb{R}$. 
Among the implications

$$
\left(\mathrm{I}^{\prime}\right) \Rightarrow(\mathrm{I}) \Rightarrow(\mathrm{II}) \Rightarrow\left(\mathrm{II}^{\prime}\right) \Rightarrow\left(\mathrm{III}^{\prime}\right) \Rightarrow\left(\mathrm{I}^{\prime}\right)
$$

only the first is nontrivial and it follows from [9, Lemma 1].

Remark 2. If we consider the category version of Lemma 1 (which holds true, see [1, Prop. 1.5]) and category versions of $\left(\mathrm{I}^{\prime}\right),\left(\mathrm{II}^{\prime}\right)$, and $\left(\mathrm{III}^{\prime}\right)$, Baire class 1 functions should be used in $\left(\mathrm{I}^{\prime}\right),\left(\mathrm{II}^{\prime}\right)$, and $\left(\mathrm{III}^{\prime}\right)$ instead of continuous ones, and then all the conditions are equivalent. This follows from the fact that if $F_{f}$ has the Baire property for every Baire class 1 function $f: \mathbb{R} \rightarrow \mathbb{R}$ then $F$ is Baire sup-measurable. To show this, consider a function $g: \mathbb{R} \rightarrow \mathbb{R}$ with the Baire property. Then $g \mid A$ is continuous for a $G_{\delta}$ comeager set $A \subseteq \mathbb{R}$. We can extend $g$ to a function $f: \mathbb{R} \rightarrow \mathbb{R}$ of Baire class $1[11, \S 35, \mathrm{VI}]$. Thus $F_{g}(x)=F_{f}(x)$ for each $x \in A$. Hence $F_{g}$ has the Baire property.

Remark 3. Observe that there is an $F: \mathbb{R}^{2} \rightarrow \mathbb{R}$ such that $F_{f}$ has the Baire property for each continuous $f: \mathbb{R} \rightarrow \mathbb{R}$ but $F$ is not Baire sup-measurable.

To see it, first notice that the existence of an $F$ as above follows from the existence of a continuous function $g:[0,1] \backslash D \rightarrow \mathbb{R}$, where $D$ is the set of all dyadic numbers, such that

$$
\text { the set }\{x \in[0,1] \backslash D: f(x)=g(x)\} \text { is nowhere dense }
$$

for every continuous $f: \mathbb{R} \rightarrow \mathbb{R}$.

Indeed, if $g$ is such a function, take a subset $B$ of $[0,1] \backslash D$ without the Baire property and let $F: \mathbb{R}^{2} \rightarrow \mathbb{R}$ be the characteristic function of $g \mid B$. Then $F$ is not Baire sup-measurable since $F_{\bar{g}}^{-1}[\{1\}]=B$, where $\bar{g}: \mathbb{R} \rightarrow \mathbb{R}$ is a Borel extension of $g$. On the other hand $F_{f}$ has the Baire property for every continuous function $f: \mathbb{R} \rightarrow \mathbb{R}$ since, by (5), there exists a nowhere dense set $N$ such that $F_{f}(x)=0$ for all $x \in \mathbb{R} \backslash N$.

Now, function $g$ satisfying (5) is constructed as follows. For $x \in[0,1] \backslash D$ let $\left\langle i_{n}(x) \in\{0,1\}: n<\omega\right\rangle$ be a sequence from the unique binary representation of $x$, that is, such that $x=\sum_{n<\omega} i_{n}(x) 2^{-(n+1)}$. The binary representation of $g(x)$ is obtained from the binary representation of $x$ by leaving the digits on the odd places unchanged and by interchanging all 0's and 1's at even places, that is, we put

$$
g(x)=\sum_{k<\omega} i_{2 k+1}(x) 2^{-(2 k+2)}+\sum_{k<\omega}\left(1-i_{2 k}(x)\right) 2^{-(2 k+1)} .
$$

Now, $g$ is continuous, since $\left|g(x)-g\left(x^{\prime}\right)\right|=\left|x-x^{\prime}\right|$ for any $x, x^{\prime} \in[0,1] \backslash D$. To finish the argument for (5) take continuous function $f: \mathbb{R} \rightarrow \mathbb{R}$ and put 
$N=\{x \in[0,1] \backslash D: f(x)=g(x)\}$. The set $N$ is nowhere dense since for every $d \in D$ there exists an $h>0$ such that

$$
\text { either } N \cap(d, d+h)=\emptyset \text { or } N \cap(d-h, d)=\emptyset \text {. }
$$

For suppose not, and let $y \in D$ witness for it. Then, there are sequences $h_{j} \rightarrow y$ and $k_{j} \rightarrow y$ in $N$ such that the first is increasing and the second is decreasing. Since $y \in D$, there exist sequences: $\left\langle i_{n}^{+}(y) \in\{0,1\}: n<\omega\right\rangle$ eventually equal to 0 , and $\left\langle i_{n}^{-}(y) \in\{0,1\}: n<\omega\right\rangle$ eventually equal to 1 such that $y=\sum_{n<\omega} i_{n}^{+}(y) 2^{-(n+1)}=\sum_{n<\omega} i_{n}^{-}(y) 2^{-(n+1)}$. Let

$$
g^{+}(y)=\sum_{k<\omega} i_{2 k+1}^{+}(y) 2^{-(2 k+2)}+\sum_{k<\omega}\left(1-i_{2 k}^{+}(y)\right) 2^{-(2 k+1)}
$$

and

$$
g^{-}(y)=\sum_{k<\omega} i_{2 k+1}^{-}(y) 2^{-(2 k+2)}+\sum_{k<\omega}\left(1-i_{2 k}^{-}(y)\right) 2^{-(2 k+1)} .
$$

Then

$$
\lim _{n \rightarrow \infty} f\left(h_{n}\right)=\lim _{n \rightarrow \infty} g\left(h_{n}\right)=g^{-}(y) \neq g^{+}(y)=\lim _{n \rightarrow \infty} g\left(k_{n}\right)=\lim _{n \rightarrow \infty} f\left(k_{n}\right)
$$

contradicting continuity of $f$.

We are going to study the question whether condition (I) given in Lemma 1 is independent of ZFC. However, the equivalent condition (II) will be more convenient. In the sequel it will be denoted by (Nsup). As we have mentioned before, (Nsup) holds true in some models of ZFC where $\mathrm{CH}$ or some weaker conditions (e.g. MA or $\operatorname{non}(\mathcal{N})=\mathfrak{c}$ ) are assumed. A. Rosłanowski and I. Recław (oral communication) have observed independently that a Luzin set $H \subseteq \mathbb{R}^{2}$ fulfills (Nsup). In fact, we will try to show that some versions of (Nsup), which seem stronger, are independent of ZFC. They are the following

(Nsup1) there is a nonmeasurable set $H \subseteq \mathbb{R}^{2}$ which is a function from $\mathbb{R}$ to $\mathbb{R}$ such that $\operatorname{dom}(H \cap f)$ is measurable for each Borel function $f: \mathbb{R} \rightarrow \mathbb{R}$;

(Nsup2) there is a nonmeasurable set $H \subseteq \mathbb{R}^{2}$ which is a function with a nonmeasurable domain $\operatorname{dom}(H)$ and such that $\operatorname{dom}(H \cap f)$ is measurable for each Borel function $f: \mathbb{R} \rightarrow \mathbb{R}$.

Conditions (Nsup), (Nsup1), and (Nsup2) have their category analogues and if we want to distinguish them, we write L-(Nsup) (like Lebesgue) or B-(Nsup) (like Baire), and similarly for (Nsup1) and (Nsup2). To show that (Nsup1) and (Nsup2) hold true in some models of ZFC we recall the construction from [1, 
Prop. 1.7] in a modified, sharper version. For this, we need the following definitions.

A Borel set $B \subseteq \mathbb{R}^{2}$ is called big if $\left|\left\{x \in \mathbb{R}:\left|B_{x}\right|>\omega\right\}\right|>\omega$ where $B_{x}=\{y \in \mathbb{R}:\langle x, y\rangle \in B\}$ for $x \in \mathbb{R}$. Observe that, by the Fubini theorem and its category analog, all Borel sets of positive measure and all Borel nonmeager sets in $\mathbb{R}^{2}$ are big. Also, we will say that a set $T \subseteq \mathbb{R}$ is thick provided it intersects every perfect subset of $\mathbb{R}$. (Such an intersection must then have cardinality c.) Recall that a set $S \subseteq \mathbb{R}$ is a Bernstein set if both $S$ and $\mathbb{R} \backslash S$ are thick.

Theorem 1. Assume that $T \subseteq \mathbb{R}$ is thick. Then there exists a set $H \subseteq T \times \mathbb{R}$ such that

- $\left|H_{x}\right|=1$ for each $x \in T$, that is, $H$ is a function from $T$ into $\mathbb{R}$,

- $|\operatorname{dom}(H \cap f)|<\mathfrak{c}$ for each Borel function $f: \mathbb{R} \rightarrow \mathbb{R}$,

- $H \cap B \neq \emptyset$ for each big set $B \subseteq \mathbb{R}^{2}$.

In particular $H$ is neither measurable nor has the Baire property (as a subset of $\mathbb{R}^{2}$ ).

Proof. Note that if $B \subseteq \mathbb{R}^{2}$ is a big set then $\left|B_{x}\right|>\omega$ is equivalent to $\left|B_{x}\right|=\mathfrak{c}$ since $B_{x}$ is Borel. Also $\left\{x \in \mathbb{R}:\left|B_{x}\right|>\omega\right\}$ is analytic [11, $\S 39$, VII, Th. 3] and since it is uncountable, it must contain a perfect subset. In particular,

$$
\left|\left\{x \in T:\left|B_{x}\right|>\omega\right\}\right|=\mathfrak{c} .
$$

That will be used in the construction.

First, we arrange, respectively, all numbers from $T$, all big subsets of $\mathbb{R}^{2}$, and all Borel functions from $\mathbb{R}$ to $\mathbb{R}$, into one-to-one transfinite sequences

$$
\begin{aligned}
& \left\langle x_{\alpha}: \alpha<\mathfrak{c}\right\rangle, \\
& \left\langle B_{\alpha}: \alpha<\mathfrak{c}\right\rangle, \\
& \left\langle f_{\alpha}: \alpha<\mathfrak{c}\right\rangle .
\end{aligned}
$$

For $\alpha<\mathfrak{c}$ we will define recursively the real numbers $s_{\alpha}, y_{\alpha}, t_{\alpha}$, and $z_{\alpha}$ as follows. Let $s_{0}=x_{0}$ and pick an arbitrary $y_{0} \in \mathbb{R}$. Let $t_{0}$ be the first number in (7) different from $s_{0}$ and such that $\left(B_{0}\right)_{t_{0}} \neq \emptyset$. Then pick an arbitrary $z_{0} \in\left(B_{0}\right)_{t_{0}}$.

Next, assume that $0<\beta<\mathfrak{c}$ and that the points $s_{\alpha}, y_{\alpha}, t_{\alpha}$, and $z_{\alpha}$ are already constructed for all $\alpha<\beta$. We will define $s_{\beta}, y_{\beta}, t_{\beta}$, and $z_{\beta}$. So, let $s_{\beta}$ be the first number in (7) different from all $s_{\alpha}$ and $t_{\alpha}$ for $\alpha<\beta$ and pick

$$
y_{\beta} \in \mathbb{R} \backslash\left\{f_{\alpha}\left(s_{\beta}\right): \alpha<\beta\right\} .
$$


Choose $t_{\beta}$ as the first number in (7) different $s_{\beta}$ and from all $s_{\alpha}$ and $t_{\alpha}$ for all $\alpha<\beta$ and such that $\left|\left(B_{\beta}\right)_{t_{\beta}}\right|=\mathfrak{c}$. Such a point exists by (6). Then pick

$$
z_{\beta} \in\left(B_{\beta}\right)_{t_{\beta}} \backslash\left\{f_{\alpha}\left(t_{\beta}\right): \alpha<\beta\right\} .
$$

This finishes the recursive construction of numbers $s_{\alpha}, y_{\alpha}, t_{\alpha}$, and $z_{\alpha}$ for all $\alpha<\mathfrak{c}$. Now, we put

$$
H=\left\{\left\langle s_{\alpha}, y_{\alpha}\right\rangle: \alpha<\mathfrak{c}\right\} \cup\left\{\left\langle t_{\alpha}, z_{\alpha}\right\rangle: \alpha<\mathfrak{c}\right\} .
$$

It follows from the construction that $\bigcup_{\alpha<\mathfrak{c}}\left\{s_{\alpha}, t_{\alpha}\right\}=T$ and that $H$ meets every big set $B_{\alpha}$. It is also clear that $\left|H_{x}\right|=1$ for each $x \in T$. Moreover, for each $\alpha<\mathfrak{c}$ we have

$$
\operatorname{dom}\left(H \cap f_{\alpha}\right) \subseteq\left\{s_{\gamma}: \gamma<\alpha\right\} \cup\left\{t_{\gamma}: \gamma<\alpha\right\} .
$$

Hence $\left|\operatorname{dom}\left(H \cap f_{\alpha}\right)\right|<\mathfrak{c}$.

Finally, if we suppose that $H$ is measurable then, by the Fubini theorem, $H$ should be a null set. But then there is a big set (of full measure) outside $H$ which contradicts our construction. Similarly $H$ does not possess the Baire property.

Corollary 1. Let $H$ be a set from Theorem 1 used with $T=\mathbb{R}$.

(a) If $\operatorname{non}(\mathcal{N})=\mathfrak{c}$ then $H$ witnesses L-(Nsup1).

(b) If $\operatorname{non}(\mathcal{M})=\mathfrak{c}$ then $H$ witnesses $\mathrm{B}-(\mathrm{Nsup} 1)$.

Corollary 2. Let $H$ be a set from Theorem 1 used with a Bernstein set $T$.

(a) If $\operatorname{non}(\mathcal{N})=\mathfrak{c}$ then $H$ witnesses L-(Nsup2).

(b) If $\operatorname{non}(\mathcal{M})=\mathfrak{c}$ then $H$ witnesses $\mathrm{B}-(\mathrm{Nsup} 2)$.

Remark 4. In the construction given in Theorem 1 we can ensure that $y_{\beta}$ and $z_{\beta}$ are chosen so that the points $\left\langle s_{\beta}, y_{\beta}\right\rangle$ and $\left\langle t_{\beta}, z_{\beta}\right\rangle$ are not colinear with any two of points from $H$ that are constructed earlier. Thus our set meets every straight line in at most two points and has all nice applications described in [9] and mentioned above.

Remark 5. By Remark 1 we may use only continuous functions $f$ in the measure formulation of (Nsup1) and (Nsup2). For category, by Remark 2, we may use functions $f$ of Baire class 1 .

Now we shall prove a characterization of (Nsup2) connected with the discontinuity of restricted functions. 
Theorem 2. Condition L-(Nsup2) is true if and only if

(*) there exists a nonmeasurable set $H \subseteq \mathbb{R}^{2}$ which is a function with a nonmeasurable domain $\operatorname{dom}(f)$ and such that for each $Z \subseteq \operatorname{dom}(H)$, with $Z \notin \mathcal{N}$, the function $H \mid Z$ is not continuous.

The analogous characterization in the category case is also true.

Proof. First we will show that L-(Nsup2) implies the condition $(*)$. So, let $H \subseteq \mathbb{R}^{2}$ satisfy L-(Nsup2) and put $S=\operatorname{dom}(H)$. Consider two cases.

Case 1. The only measurable subsets of $S$ are null sets. (In other words, the measurable kernel of $S$ is empty.) Suppose the assertion is false. Thus there is a set $Z \subseteq S, Z \notin \mathcal{N}$, such that the function $H \mid S$ is continuous. Consider a Borel extension $f: \mathbb{R} \rightarrow \mathbb{R}$ of $H \mid Z$. (See $[11$, Th. 1, $\S 35, \mathrm{I}]$.) Then $Z \subseteq \operatorname{dom}(f \cap H) \subseteq S$, so $\operatorname{dom}(f \cap H)$ cannot be measurable, a contradiction.

Case 2. The measurable kernel $K$ of $S$ is nonempty. Observe that then $H^{*}=H \backslash(K \times \mathbb{R})$ can play the role of $H$ in L-(Nsup2) and thus Case 1 works. So, there is a set $Z \subseteq S \backslash K, Z \notin \mathcal{N}$, such that the function $H^{*}|Z=H| Z$ is continuous.

The proof of the category version of this implication is essentially the same.

Now, to prove that $(*)$ implies L-(Nsup2) let $H \subseteq \mathbb{R}^{2}$ satisfy $(*)$ and suppose that L-(Nsup2) is false. Thus there is a continuous function $f: \mathbb{R} \rightarrow \mathbb{R}$ such that $\operatorname{dom}(H \cap f)$ is nonmeasurable. (Compare Remark 5.) Obviously for $Z=\operatorname{dom}(H \cap f)$ the function $f \mid Z$ is continuous. Thus $H \mid Z$ is continuous, a contradiction.

In the category case we proceed similarly. Namely, we suppose that there is a function $f: \mathbb{R} \rightarrow \mathbb{R}$ with the Baire property such that $\operatorname{dom}(H \cap f)$ does not have the Baire property. Hence there exists a comeager $A \subseteq \mathbb{R}$ such that $f \mid A$ is continuous. Thus $f \mid Z$ is continuous for $Z=A \cap \operatorname{dom}(H \cap f) \notin \mathcal{M}$, a contradiction.

A function $g$ from $S \subseteq \mathbb{R}$ to $\mathbb{R}$ will be called ugly if $S$ is nonmeasurable and $g$ is nonmeasurable as a subsets of $\mathbb{R}^{2}$. Thus, by Theorem 2, condition $\neg(\mathrm{L}-(\mathrm{Nsup} 2))$ is equivalent to the following statement.

For every ugly function $g$ there exists a set $Z \subseteq \operatorname{dom}(g), Z \notin \mathcal{N}$, such that $g \mid Z$ is continuous.

The category case is analogous.

A similar statement for functions $g: \mathbb{R} \rightarrow \mathbb{R}$ is connected with the following open problem of von Weizsäcker. (See [5, Problem AR(a)] or [4, Problem 1].)

Is it consistent that every function $g: \mathbb{R} \rightarrow \mathbb{R}$ restricted to some set of positive outer measure is continuous? 
The property considered in the above question will be denoted by (vWH), that is,

(vWH) For every function $g: \mathbb{R} \rightarrow \mathbb{R}$ there exists a set $Z \notin \mathcal{N}$ such that $g \mid Z$ is continuous.

Remark 6. From (vWH) it follows that the same statement is true provided $g: S \rightarrow \mathbb{R}$ and $S \subseteq \mathbb{R}$ is an $F_{\sigma}$ set such that $S \notin \mathcal{N}$.

Indeed, each of the spaces $\mathbb{R}$ and $S$ can be partitioned into a countable number of uncountable Polish spaces with nonzero finite Lebesgue measure. In the case of $S$ we consider int $S$ (the interior of $S$ ) and divide $S \backslash \operatorname{int} S$ into a countable number of closed parts. Next, if necessary, we divide int $S$ into uncountable parts and add to them countable parts from the division of $S \backslash \operatorname{int} S$. Then we consider a Borel isomorphism $\varphi$ from $\mathbb{R}$ onto $S$ which transforms Polish parts of $\mathbb{R}$ onto the respective Polish parts of $S$ and such that for each $E \subseteq \mathbb{R}$ the conditions $E \in \mathcal{N}$ and $\varphi[E] \in \mathcal{N}$ are equivalent. This can be deduced from the fact that any two Polish probability spaces (where the probabilities vanish on the singletons) are Borel isomorphic. (See e.g. [2, Th. 4.20].) Now, if $g: S \rightarrow \mathbb{R}$, we apply (vWH) to $g \circ \varphi$ and find a set $Z \notin \mathcal{N}$ such that $(g \circ \varphi) \mid Z$ is continuous. Thus $\varphi[Z] \notin \mathcal{N}$. By the Luzin theorem we find a sequence $\left\{F_{n}\right\}_{n=1}^{\infty}$ of pairwise disjoint closed subsets of $S$ of positive measure such that $S \backslash \bigcup_{n=1}^{\infty} F_{n} \in \mathcal{N}$ and $\varphi^{-1} \mid F_{n}$ is continuous for every $n$. Since for the outer Lebesgue measure $\lambda^{*}$ we have

$$
\lambda^{*}(\varphi[Z])=\sum_{n=1}^{\infty} \lambda^{*}\left(\varphi[Z] \cap F_{n}\right),
$$

therefore $W=\varphi[Z] \cap F_{k} \notin \mathcal{N}$ for some $k$ and $\varphi^{-1} \mid W$ is continuous. Thus $\left(g \circ \varphi \circ \varphi^{-1}\right)|W=g| W$ is continuous.

Remark 7. The category analogue of ( $\mathrm{vWH})$ is consistent with $\mathrm{ZFC}$ which was shown by Shelah [12].

Remark 8. From the category analogue of (vWH) it follows its version where $g: S \rightarrow \mathbb{R}$ and $S \subseteq \mathbb{R}$ is a $G_{\delta}$ set such that $S \notin \mathcal{M}$.

The proof is similar to that presented for Remark 6 . We use the fact that there is a Borel isomorphism $\varphi$ from $\mathbb{R}$ onto $S$ such that for each $E \subseteq \mathbb{R}$ the conditions $E \in \mathcal{M}$ and $\varphi[E] \in \mathcal{M}$ are equivalent. (See e.g. [2, Th. 3.15].) If $g: S \rightarrow \mathbb{R}$, we find a set $Z \notin \mathcal{M}$ such that $(g \circ \varphi) \mid Z$ is continuous. Hence $\varphi[Z] \notin \mathcal{M}$. Note that $\varphi^{-1} \mid A$ is continuous for a comeager subset $A$ of $S$. Thus $W=\varphi[Z] \cap A \notin \mathcal{M}$ and $\left(g \circ \varphi \circ \varphi^{-1}\right)|W=g| W$ is continuous. 
Theorem 3. Condition (vWH) implies condition $\neg(\mathrm{L}-(\mathrm{Nsup} 1))$. Moreover, the analogous implication for the category case is also true.

Proof. We have to show that for each function $H: \mathbb{R} \rightarrow \mathbb{R}$ which is a nonmeasurable subset of $\mathbb{R}^{2}$ there exists a Borel function $f: \mathbb{R} \rightarrow \mathbb{R}$ such that $\operatorname{dom}(H \cap f)$ is nonmeasurable. Thus let $H: \mathbb{R} \rightarrow \mathbb{R}$ be a function such that $H \subseteq \mathbb{R}^{2}$ is nonmeasurable. Applying (vWH) to $g=H$ we obtain a set $Z \notin \mathcal{N}$ such that $H \mid Z$ is continuous. Consider a Borel extension $f: \mathbb{R} \rightarrow \mathbb{R}$ of $H \mid Z$. Then $\operatorname{dom}(H \cap f) \notin \mathcal{N}$. If $\operatorname{dom}(H \cap f)$ is nonmeasurable, the proof is finished. So assume that $\operatorname{dom}(H \cap f)$ is measurable and include it in a $G_{\delta}$ set $A$ of the same measure. Let $B=\mathbb{R} \backslash A$. Then $B \notin \mathcal{N}$ since otherwise $H=f$

almost everywhere, and thus $H$ would be a measurable subset of $\mathbb{R}^{2}$. Now, we apply the version of $(\mathrm{vWH})$ from Remark 6 to the function $H \mid B$. Then there is a set $Z_{1} \subseteq B, Z_{1} \notin \mathcal{N}$, such that $H \mid Z_{1}$ is continuous. Consider a Borel extension $f_{1}: B \rightarrow \mathbb{R}$ of $H \mid Z_{1}$. Then $\operatorname{dom}\left(H \cap f_{1}\right) \notin \mathcal{N}$. If $\operatorname{dom}\left(H \cap f_{1}\right)$ is nonmeasurable, the proof is finished since, for the Borel function $h: \mathbb{R} \rightarrow \mathbb{R}$ given by

$$
h(x)= \begin{cases}f(x) & \text { for } x \in A \\ f_{1}(x) & \text { for } x \in B\end{cases}
$$

the set $\operatorname{dom}(H \cap h)$ is nonmeasurable. So assume that $\operatorname{dom}\left(H \cap f_{1}\right)$ is measurable and include it in a $G_{\delta}$ set $A_{1}$ of the same measure. Let $B_{1}=B \backslash A_{1}$. Then $B_{1} \notin \mathcal{N}$ since otherwise, $H=h$ almost everywhere (for $h$ given above), and thus $H$ would be a measurable subset of $\mathbb{R}^{2}$. We proceed inductively, constructing the sets $A_{\xi}$ and $B_{\xi}$ for $\xi<\omega_{1}$. In some step before $\omega_{1}$ we obtain the required Borel function since otherwise, the sets $A, A_{1}, A_{2}, \ldots, A_{\xi}, \ldots$ would exhaust $\mathbb{R}$ in a countable number of steps (that is, their union would be of full measure in $\mathbb{R}$ ) and consequently $H$ would be a measurable subset of $\mathbb{R}^{2}$.

In the analogous proof for the category analog we use Remark 8.

From Remark 7 and the category part of Theorem 3 we infer the following.

Corollary 3. $\neg(\mathrm{B}-(\mathrm{Nsup} 1))$ is consistent with ZFC.

From Corollaries 1(b) and 3 we obtain also

Corollary 4. B-(Nsup1) is independent of ZFC.

\section{References}

[1] M. Balcerzak, Some remarks on sup-measurability, Real Anal. Exchange 17 (1991-92), 597-607. 
[2] J. Cichoń, A. B. Kharazishvili, B. Wȩglorz, Subsets of the Real Line, Łódź Academic Press, Łódź 1995.

[3] K. Ciesielski, Set Theory for the Working Mathematician, London Math. Soc. Student Texts 39, Cambridge Univ. Press 1997.

[4] K. Ciesielski, Set theoretic real analysis, J. Appl. Anal. 3(2) (1997), 143190. (Preprint available in the electronic form from the Set Theoretic Analysis Web Page:

http://www.math.wvu.edu/homepages/kcies/STA/STA.html)

[5] D. H. Fremlin, Problems, circulated notes (1996).

[6] E. Grande, Z. Grande, Quelques remarques sur la superposition $F(x, f(x))$, Fund. Math. 121 (1984), 199-211.

[7] Z. Grande, J. Lipiński, Un example d'une fonction sup-mesurable qui n'est pas mesurable, Colloq. Math. 39 (1978), 77-79.

[8] A. B. Kharazishvili, Some questions from the theory of invariant measures, Bull. Acad. Sci. Georgian SSR 100 (1980) (in Russian).

[9] A. B. Kharazishvili, Sup-measurable and weakly sup-measurable mappings in the theory of ordinary differential equations, J. Appl. Anal. 3(2) (1997), 211-223.

[10] M. A. Krasnosel'skiı̌, A. V. Pokrovskiı̌, Systems with Hysteresis, Springer, Berlin, 1988.

[11] K. Kuratowski, Topology, vol.1, Academic Press, New York 1966.

[12] S. Shelah, Possibly every real function is continuous on a non-meagre set, Publ. Inst. Mat. (Beograd) (N.S.) 57 (1995), 47-60.

[13] J. W. S̆ragin, Conditions for measurability of superpositions, Dokl. Akad. Nauk SSSR 197 (1971), 295-298 (in Russian). 